\title{
Acute Pancreatitis Associated with Lamivudine Therapy for Chronic B Hepatitis
}

\author{
Felipe Francisco Tuon ${ }^{1}$, Cristina Maria de Fátima Guastini ${ }^{2}$ and Maria Ivete Castro Boulos ${ }^{1}$ \\ ${ }^{1}$ Department of Infectious Diseases, University of São Paulo, Medical School; ${ }^{2}$ Infectious and Parasitic Diseases Clinic, Hospital das Clinicas, \\ Medical School, University of São Paulo; São Paulo, SP, Brazil
}

\begin{abstract}
Hyperamylasemia is a common complication during lamivudine use. We report a case of a pancreatitis following lamivudine therapy. A careful monitoring of amylase levels during treatment with lamivudine is discussed, mainly in the first weeks, considering the cost of this exam and further complication.

Key-Words: Lamivudine, pancreatitis, amylases, hyperamilasemina, hepatitis, chronic B hepatitis.
\end{abstract}

Side effects associated with lamivudine therapy are rarely described. Gastrointestinal symptoms, such as nausea, vomiting, diarrhea, and abdominal pain have been described, though more severe conditions can occur, such as rhabdomyolysis and severe anemia [1]. Hyperamylasemia is a well-known complication after lamivudine use, and serum concentrations of amylase after 6 to 12 months of treatment are higher than those before treatment [2]. These side effects are most commonly described in patients with HIV infection than in those with chronic B hepatitis. We report a case of pancreatitis following three weeks of lamivudine therapy, without severe complications. A complication of lamivudine treatment in a patient with chronic B hepatitis is extremely rare, and physicians do not normally associate lamivudine with side effects.

A 59-year-old man with a history of five years of chronic B hepatitis (Anti-HBe and HBsAg positive) initiated treatment with $150 \mathrm{mg}$ lamivudine on February 2007. This drug was indicated because anatomopathological findings showed intense periportal inflammation (grade 4) and increased alanine aminotransferase (ALT) levels (195 U/L). The patient was asymptomatic, so other exams were made: $\mathrm{Hb}-15.6 \mathrm{~g} /$ $\mathrm{dL}$; leukocytes $-6,590 / \mathrm{mm}^{3}$; platelets $-164,000 / \mathrm{mm}^{3}$; prothrombin time $-13,5$ seconds (INR -1.1 ); aspartate aminotransferases (AST) $-100 \mathrm{U} / \mathrm{L}$; total bilirubin -0.8 $\mathrm{mg} / \mathrm{dL}$; total protein $-7.6 \mathrm{~g} / \mathrm{dL}$ (albumin $-4.8 \mathrm{~g} / \mathrm{dL}$ ); serum protein electrophoresis showed $23.4 \%$ gamma globulines $(1.8 \mathrm{~g} / \mathrm{dL})$. Renal function was normal.

Fifteen days after beginning on lamivudine, the patient was admitted to the hospital with intense abdominal pain, nausea and vomiting, anorexia and malaise. Serum exams revealed hyperamylasemia (778 U/ $\mathrm{L})$, increased lactate dehydrogenase $(233 \mathrm{U} / \mathrm{L}$, normal = 190U/L). Leukocytosis $\left(14,200 / \mathrm{mm}^{3}\right)$ was also found. An Received on 12 January 2008; revised 14 May 2008. Address for correspondence: Dr. Felipe Francisco Tuon Department of Infectious Diseases, University of São Paulo, Medical School, São Paulo, Brazil. Avenida Dr. Enéas de Carvalho Aguiar,470, Cerqueira César. Zip code: 05403-000. São Paulo, SP, Brazil. Telephone: 5511 30696530. Fax: 5511 30697508. E-mail: ffbt@pop.com.br. abdominal echography was normal, and computed tomography revealed a discrete peripancreatic edema, without signs of necrosis. After five days of observational treatment and drug interruption the patient had a good evolution, with normalization of laboratorial findings and absence of symptoms.

Lamivudine is an oral nucleoside analog that inhibits HBV replication and markedly normalizes alanine transaminase levels. This effect is directly associated with improvement in liver inflammation and necrosis activity. As side effects are rarely described, laboratory monitoring of markers, such as amylase, is not common. A previous study demonstrated that lamivudine can be associated with hyperamylasemia, but this increase was not associated with abdominal pain [3]. We have demonstrated here that hyperamylasemia can also involve abdominal pain and other clinical signs/ symptoms of pancreatitis, including discrete imaging abnormalities. In earlier and larger studies, abdominal discomfort was not different from what was found in the placebo group, and no acute pancreatitis was described [4]. There are only two previous publications indicating that lamivudine induces pancreatitis [2,5]. We conclude that amylase elevation can be found in patients with chronic $\mathrm{B}$ hepatitis being treated with lamivudine; however, this elevation is not always benign. We also suggest careful monitoring of amylase levels during treatment with lamivudine, mainly in the first weeks, taking into account the cost of this exam and of further complications.

\section{References}

1. Adani G.L., Baccarani U., Risaliti A., et al. Rhabdomyolysis due to Lamivudine administration in a liver transplant recipient. Am J Transplant 2005;5:634.

2. Chen C.H., Changchien C.S., Lu S.N., et al. Lamivudine treatment for recurrent pancreatitis associated with reactivation of chronic B hepatitis. Dig Dis Sci 2002;47:564-7.

3. Ertekin V., Selimoglu M.A., Orbak Z. Effects of lamivudine therapy on the glucose metabolism in children with chronic hepatitis B: first year follow-up results. Eur J Gastroenterol Hepatol 2005; 17:655-9.

4. Dienstag J.L., Schiff E.R., Wright T.L., et al. Lamivudine as initial treatment for chronic hepatitis B in the United States. N Engl J Med 1999;341:1256-63.

5. Soylu A.R., Dokmeci G., Tezel A., et al. Lamivudine-induced acute pancreatitis in a patient with decompensated Hbvrelated chronic liver disease. J Clin Gastroenterol 2004;38:134. 